\title{
Perioperative Management of a Patient with C1 Esterase Deficiency and a Known Difficulty Airway, a Case Report with Mini Reviews
}

\author{
Jonathan A. Bond, D.O, M.P.H ${ }^{1}$, Dylan T. Anderson, B.S ${ }^{2}$, Hong Wang, M.D, Ph.D ${ }^{1^{*}}$ \\ ${ }^{1}$ Department of Anesthesiology, West Virginia University, Morgantown, West Virginia, United States of America \\ ${ }^{2}$ School of Medicine, West Virginia University, Morgantown, West Virginia, United States of America
}

\begin{abstract}
Hereditary angioedema (HAE) results from an underlying absence or dysfunction of the $\mathrm{C} 1$ esterase inhibitor and concurrent exposure to stressors, including surgery. Type I and II are caused by a mutation in the C1NH gene. Type I is due to deficiency of the C1 inhibitor; Type II is due to the dysfunction of the $\mathrm{C} 1$ inhibitor. This results in diffuse swelling and possible airway catastrophe, which can persist when incorrectly treated as anaphylaxis or allergic angioedema. We report the successful management of a HAE patient with a previously identified difficult airway undergoing elective surgery.
\end{abstract}

\section{Keywords}

C1 esterase inhibitor, airway management, hereditary angioedema, elective surgery

\section{Abbrevations \\ $\mathrm{HAE}=$ hereditary angioedema; $\mathrm{PAU}=$ pre-admission unit}

\section{Introduction}

Hereditary angioedema (HAE) is characterized by the absence or dysfunction of the $\mathrm{C} 1$ esterase inhibitor of the complement pathway [1]. Often stimulated by stressors including surgery, this causes an unregulated release of bradykinin, triggering an inflammatory cascade that results in extensive edema [2]. As a result, C1 esterase inhibitor deficiency can result in an unrecognized complicated airway.

We report a case summarizing the management of a patient with HAE and a known difficult airway. The patient provided written consent to write and publish this case report.

\section{Case Description}

A 54-year old, previously healthy female presented to an academic medical center in rural Appalachia for elective surgery. Her past medical history was significant for hypothyroidism, anxiety, depression, and C1 esterase inhibitor deficiency (Type I) diagnosed as a child after frequent episodes of intractable abdominal pain and resultant angioedema. She was unable to identify any particular triggers as the previous attacks occurred spontaneously. Past surgical history was significant for a previous difficult intubation at an outside facility during an endoscopic retrograde cholangiopancreatography procedure. The anesthesia records were unable to be obtained; however, the patient stated this was not precipitated by an episode of angioedema.

The patient's BMI was $28.5 \mathrm{~kg} / \mathrm{m}^{2}$ and her airway consisted of a Mallampati 3 classification. The thyromental distance was $<6 \mathrm{~cm}$, and she displayed poor mouth opening with an inter incisor distance of $<3 \mathrm{~cm}$.

The patient was evaluated in our pre-admission unit (PAU) by a faculty anesthesiologist. The patient's HAE medical regimen included 4,000 units of $\mathrm{C} 1$ inhibitor bi-weekly, and a rescue bradykinin inhibitor, which she had not required to date. She was advised to bring both the $\mathrm{C} 1$ and bradykinin inhibitor with her on the day of surgery, as these medications are extremely expensive and are not routinely stored in our pharmacy.

Prophylactic recommendations published by Williams and Craig [3] were utilized, in which 1,000 units of $\mathrm{C} 1$ inhibitor were administered in the preoperative area one hour prior to surgery. Two doses of the bradykinin inhibitor were readily available for treatment of an attack. Awake fiberoptic intubation was not attempted due to the patient's anxiety and anticipated easy mask ventilation. However, the difficult airway cart, a video laryngoscope, and bronchoscope were immediately available. She was administered $100 \mathrm{mg}$ of hydrocortisone and pre-medicated with $2 \mathrm{mg}$ of midazolam prior to moving to the operating room.

She was intravenously induced with $100 \mu \mathrm{g}$ of fentanyl, $100 \mathrm{mg}$ of lidocaine, and $100 \mathrm{mg}$ of Propofol. A small dose of rocuronium was administered to facilitate the intubation.

The patient was successfully intubated by video la- 
ryngoscopy. To reduce the risk of airway stimulation, a smaller sized ( $6.5 \mathrm{~mm}$ diameter) endotracheal tube was smoothly advanced through the vocal cords with appropriate end tidal carbon dioxide, chest rise and bilateral breath sounds, confirming appropriate placement.

The patient was maintained with $2 \%$ sevoflurane, and the remainder of the case was uneventful. The patient was reversed with sugamadex, a train of four ratio of $>0.9$ with sustained tetanus was achieved and sufficient tidal volumes $>4 \mathrm{ml} / \mathrm{kg}$ were acquired. The patient followed commands and was extubated to 10 liters per minute of oxygen via facemask and transported to the recovery room.

After meeting recovery milestones, the patient was sent to the step-down unit for post-operative monitoring. The patient did not develop any episodes of abdominal pain or angioedema. There were no immediate complications and she was discharged 24 hours post-operatively.

\section{Discussion and Mini Review}

Hereditary angioedema is associated with mutations in the SERPING1 gene causing either absence (Type I) or alterations (Type II) of the $\mathrm{C} 1$ inhibitor, the crucial regulatory protein of the bradykinin-generating process. A C1 defect causes accumulation of bradykinin, resulting in angioedema attacks. In managing bradykinin mediated HAE, it is important to be aware of prodromal symptoms as these can be prognostic. In a study performed at the US HAEA Angioedema Center, 95.3\% of bradykinin mediated HAE patients experienced prodromal symptoms which were often predictive of an impending attack [4].

HAE is rarely encountered during anesthesia, with a prevalence of only 1 in 50,000 individuals [5]. Consensus guidelines were updated in 2017 for the general management of patients with HAE [6]. For pre-operative treatment, most HAE patients respond well to the administration of 1,000 units or $20 \mathrm{u} / \mathrm{kg}$ of $\mathrm{C} 1$ inhibitor [6]. In a rural setting or if $\mathrm{C} 1$ inhibitor is not available, FFP [7] and attenuated androgens [8] are available as second-line options. When using attenuated androgens, recommendations include administering for 5 days before and 2-3 days after surgery [8].

During any procedure, there should be at least two treatment doses available at all time [8]. Concentrated C1 inhibitor, Ecallantide and Icatibant, are recommended as first line therapy. Ecallantide is a kallikrein inhibitor used to treat HAE attacks in patients above 12 years of age, while Icatibant is a bradykinin-receptor antagonist, only available to patients above the age of 18 [6]. We used the pharmacologic recommendations published by Williams and Craig [3] for short-term prophylaxis. Specifically, the patient's prescribed C1 inhibitor was administered prophylactically, and ultimately helped in preventing an episode of angioedema throughout the perioperative period.

There is a rare subset of SERPING1 mutations that are defined as non-pathogenic and result in normal C1q complex levels $30 \%$ of the time [9]. This group may not respond to typical treatment. In a case report by Rasmussen and colleagues, these patients were found to produce C1 inhibitor autoantibodies [9]. However, prophylactic treatment with attenuated androgens was shown to prevent HAE episodes from occurring [9].

While experts suggest avoiding airway manipulation altogether in those with $\mathrm{C} 1$ esterase inhibitor deficiency [3], patient risk factors and the case type often mandate endotracheal intubation. There are no specific contraindications to induction medications, volatile agents, or paralytics in HAE [10]. One must be cautious when managing the airway of an HAE patient, however, as irritation can trigger an angioedema attack. In a case report by Maynard and colleagues, a 66-year-old male successfully underwent an urgent laparoscopic partial gastrectomy without angioedema [11]. They recommend utilizing a video laryngoscope and minimizing IV fluids to reduce the potential for airway swelling [11].

Our team discussed the risks and benefits of reversal agents, including concerns for weakness, allergic reactions, and further airway compromise. A review by MacBeth and colleagues examined post-operative complications in patients with HAE [12]. While episodes of angioedema were minimal, one patient undergoing mastectomy did develop hypoxia and post-extubation stridor [12]. Although there is documented potential of allergic reactions to Sugamadex [13], the decision was made to use Sugamadex to prevent residual neuromuscular blockade and to avoid further airway manipulation (i.e., an oral airway or jaw thrust maneuver), as this alone could precipitate an acute attack. In a case by Furtado, an angioedema patient was successfully reversed with Sugamadex and discharged 8 hours post-surgery with hemodynamic stability and no angioedema [14].

Recombinant C1 inhibitor, video laryngoscopy, and novel reversal agents may not be readily accessible in a rural healthcare setting. However, the availability of these modalities contributed to a safe outcome for this patient and should be considered when performing general anesthesia for patients with HAE and a known a difficult airway. The key components of successful perioperative management include prophylactic administration of $\mathrm{C} 1$ inhibitor, avoiding airway stimulation and diligent monitoring. It is our hope that this case provides insight on patients with HAE undergoing elective surgery and the resources we utilized to treat this patient effectively. 


\section{Author Information}

Name: Jonathan A. Bond, D.O., M.P.H.

Contribution: This author managed the patient and helped write and edit the manuscript.

Name: Dylan T. Anderson, B.S.

Contribution: This author helped write and edit the manuscript.

Name: Hong Wang, M.D., Ph.D.

Contribution: This author managed the patient and helped write and edit the manuscript.

\section{Conflict of Interest: None}

\section{References}

1. Davis AE. The Pathophysiology of Hereditary Angioedema. Clinical Immunology. 2005;114(1):3-9. doi:10.1016/j. clim.2004.05.007.

2. Vilaça MJ, Coelho FM, Faísco A, et al. Anesthetic Considerations for a Patient with Hereditary Angioedema - A Clinical Case. Brazilian Journal of Anesthesiology. 2017;67(5):541543. doi:10.1016/j.bjane.2015.03.007.

3. Williams $\mathrm{AH}$, Craig TJ. Perioperative Management for $\mathrm{Pa}-$ tients with Hereditary Angioedema. Allergy and Rhinology. 2015;6(1):50-55. doi:10.2500/ar.2015.6.0112.

4. Nguyen $\mathrm{AH}$, Riedl MA. Prodromal Symptoms in Bradykinin vs. Histamine-Mediated Angioedema. The Journal of Allergy and Clinical Immunology. 2019;143(2). doi:10.1016/j. jaci.2018.12.121.

5. Bernstein JA. Update on Angioedema: Evaluation, Diagnosis, and Treatment. Allergy and Asthma Proceedings. 2011;32(6):408-412. doi:10.2500/aap.2011.32.3469.

6. Maurer M, Magerl M, Ansotegui I, et al. The International WAO/EAACI Guideline for the Management of Hereditary Angioedema-The 2017 Revision and Update. Allergy. 2018;73(8):1575-1596. doi:10.1111/all.13384.

7. Mosier M, Riddle JD. Prophylaxis and Treatment of Hereditary Angioedema with Fresh Frozen Plasma: A Synthesis and Narrative Review. Anesthesia EJournal. 2018;6(3):1217.

8. Betschel S, Badiou J, Binkley K, et al. The International/ Canadian Hereditary Angioedema Guideline. Allergy, Asthma and Clinical Immunology. 2019;15(72). doi:10.1186/ s13223-019-0376-8.

9. Rasmussen ER, Aanæs K, Jakobsen MA, et al. Acquired Complement C1 Esterase Inhibitor Deficiency in a Patient with a Rare SERPING1 Variant with Unknown Significance. British Medical Journal Case Reports. 2019;12:e231122. doi:10.1136/bcr-2019-231122.
10. Jensen NF, Weiler JM. C1 Esterase Inhibitor Deficiency, Airway Compromise, and Anesthesia. Anesthesia and Analgesia. 1998;87(2):480-488. doi:10.1097/00000539199808000-00047.

11. Maynard AA, Burger CF, Schlesinger JJ. Angioedema: Perioperative Management. SAGE Open Medical Case Reports. 2017;5. doi:10.1177/2050313×17713912.

12. Macbeth LS, Volcheck GW, Sprung J, Weingarten TN. Perioperative Course in Patients with Hereditary or Acquired Angioedema. Journal of Clinical Anesthesia. 2016;34:385391. doi:10.1016/j.jclinane.2016.05.010.

13. Miyazaki $\mathrm{Y}$, Sunaga H, Kida K, et al. Incidence of Anaphylaxis Associated with Sugamadex. Anesthesia and Analgesia. 2018;126(5):1505-1508. doi:10.1213/ ane. 0000000000002562 .

14. Furtado I, Linda F, Pica S, et al. Anesthetic Management of Late Pressure Angioedema. Brazilian Journal of Anesthesiology. 2017;67(4):422-425. doi: 10.1016/j. bjane.2016.09.004

Citation: Bond JA, Anderson DT, Wang H. Perioperative Management of a Patient with C1 Esterase Deficiency and a Known Difficulty Airway, a Case Report with Mini Review. Transl Perioper \& Pain Med 2020; 7(4):265-267

Copyright: (C) 2020 Bond JA, et al. This is an open-access article distributed under the terms of the Creative Commons Attribution License, which permits unrestricted use, distribution, and reproduction in any medium, provided the original author and source are credited.

Corresponding Author: Hong Wang, M.D., Ph.D, 1 Medical Center Drive, PO Box 8255, Morgantown, West Virginia, 26506, USA, Tel: 304-598-4122; E-mail: Hong. wang1@wvumedicine.org

Editor: Renyu Liu, MD; PhD; Professor, Department of Anesthesiology and Critical Care, Perelman School of Medicine at the University of Pennsylvania, Center of Penn Global Health Scholar, Director of Stroke 120 Special Task Force, Chinese Stroke Association, 336 John Morgan building, 3620 Hamilton Walk, Philadelphia, PA 19104, Phone: 2157461485; Fax: 2153495078; E-mail: RenYu.Liu@pennmedicine.upenn.edu

\section{Additional publication details}

Journal short name: Transl Perioper \& Pain Med

Received Date: May 12, 2020

Accepted Date: May 16, 2020

Published Date: May 21, 2020 